\title{
Copper-Mediated Late-Stage Fluorination of Arenes Using Photoredox Catalysis
}

\section{Key words}

photoredox catalysis

sulfonium salts

fluorination

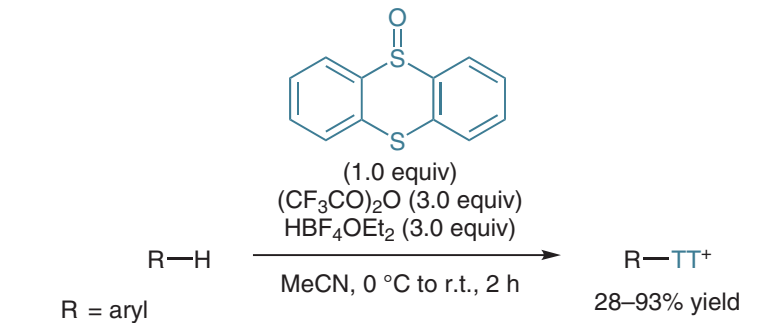

roposed mechanism:

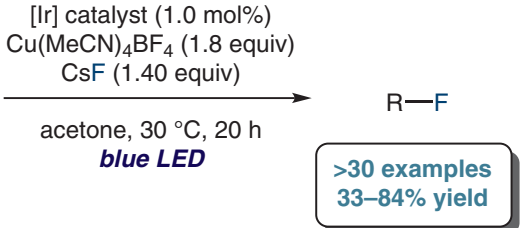

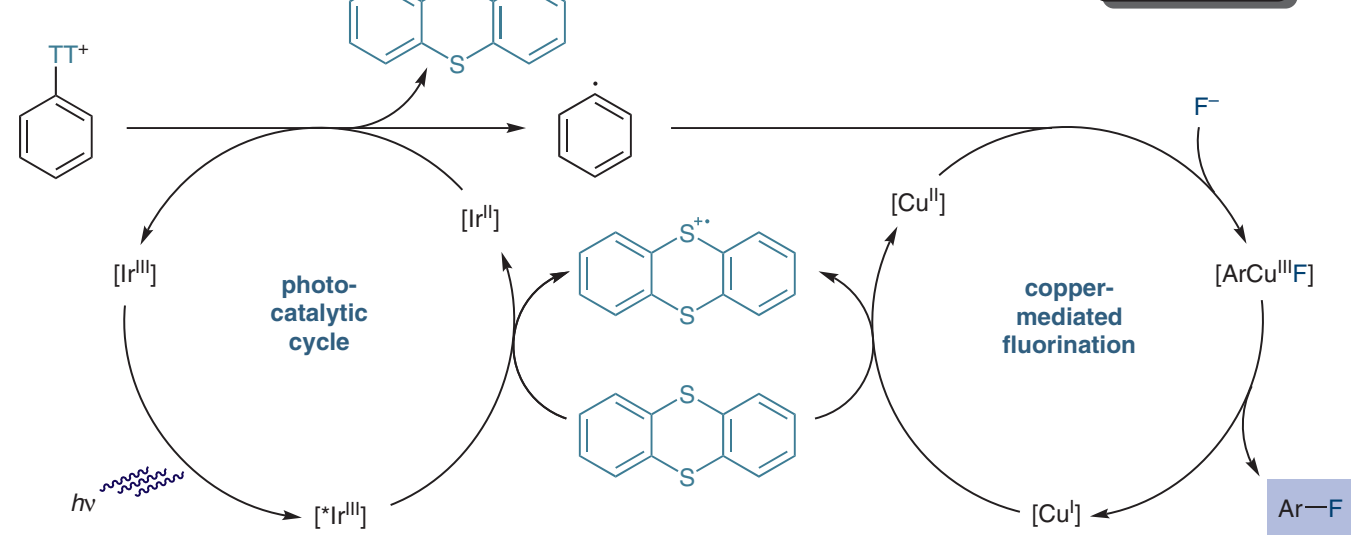

Selected examples

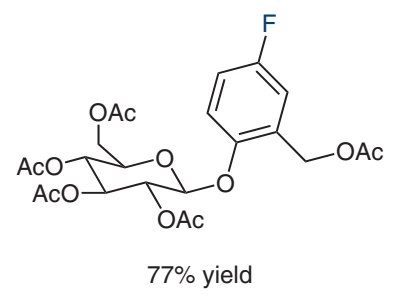

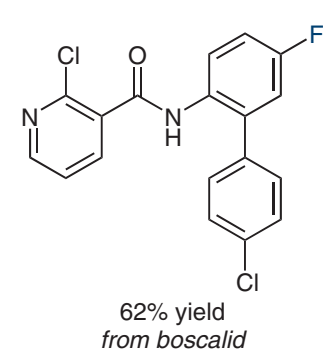

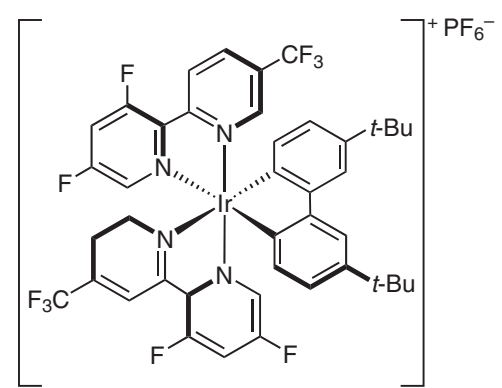

Significance: The authors report a site-selective late-stage fluorination of aryl sulfonium salts using photoredox catalysis. This newly introduced leaving group (sulfonium salt) has a relatively low redox potential and therefore provides easy access to various aryl radicals. The sulfonium group can be easily attached through thianthrenation of even complex arenes, which enables a late-stage fluorination of biological active molecules.
Comment: This method was applied to fluorinate over 30 arenes, including 12 drug-like molecules. Based on detailed experimental studies, Ritter and co-workers propose that the reaction proceeds through two perfectly meshing catalytic cycles. Therein, the sulfonium leaving group plays an important part in the recycling of both the iridium and the copper catalyst. 